\title{
INOCULACIÓN DE Citrus volkameriana Y Citrumelo swingle CON HONGOS MICORRIZÓGENOS (HMA), BAJO TÉCNICAS DIFERENTES DE DESINFECCIÓN DE SUSTRATO1
}

\author{
Susana Schweizer², Eduardo Salas 3
}

\begin{abstract}
RESUMEN
Se evaluó el efecto de la inoculación con hongos micorrizógenos sobre el crecimiento y desarrollo de dos especies de cítricos utilizados como portainjertos en la fase de semillero y al momento del trasplante a bolsas de vivero. En los años 2003 y 2004, se realizaron dos experimentos en un diseño completamente al azar con 10 repeticiones, en un vivero comercial en Alajuela, Costa Rica. Los portainjertos utilizados fueron Citrus volkameriana y Citrumelo swingle. El sustrato se desinfectó siguiendo cuatro técnicas diferentes. Se midieron la altura de la planta, el diámetro del tallo a la base, biomasa aérea, biomasa de raíces, tiempo a injertación y colonización de raíces. De los resultados obtenidos se concluye que cuando se utilizaron agroquímicos (Basamid y PCNB) para desinfectar el sustrato de vivero, se afectó negativamente la simbiosis micorrícica. Las técnicas de desinfección naturales (agua caliente y solarización) fueron más amigables con la relación mutualista. Se obtuvo una respuesta significativa de la inoculación en todas las variables medidas cuando se inoculó con una población nativa de HMA. La mejor fecha para inocular fue al momento de realizar el semillero de Citrus volkameriana y Citrumelo swingle. Los árboles inoculados con población nativa acortaron de dos a tres meses su "período a injertación", comparados con los no inoculados; de esta manera, se logró un ahorro en los gastos de mantenimiento de más de un $20 \%$. Además con este tratamiento se obtuvieron plantas más uniformes y con mejor desarrollo.
\end{abstract}

Palabras claves: micorrizas, cítricos, sustratos, desinfección.

\section{INTRODUCCIÓN}

En Costa Rica se cultivan cítricos en las regiones Huetar Norte, Chorotega, Brunca y Central. En 1998 había en el país aproximadamente 25.000 ha sembradas con naranja $\mathrm{y}$, de acuerdo con el último censo, estarían distribuidas entre más de 5.000 productores (Hernández 2000). El mismo autor menciona además que el programa de fertilización representa un $25 \%$ del costo de producción.

A nivel de vivero y para fines comerciales los cítricos se reproducen por medio de injer- to, con la finalidad de obtener producción temprana, mayor tolerancia a enfermedades y plantaciones más uniformes. En estas condiciones, los sustratos de desarrollo se desinfectan para proteger las plántulas de patógenos, pero de esta forma se eliminan también los hongos benéficos (como los HMA), lo que repercute en un pobre desarrollo de la planta.

La efectividad de la inoculación con HMA en el crecimiento de los frutales se ha demostrado en varios estudios (Chacón y Cuenca 1998; Alarcón y Ferrera-Cerrato 1999); entre los frutales, los cítricos se consideran alta-

1 Trabajo parte del Proyecto F 19-03, con financiamiento de FITTACORI.

2 Instituto Nacional de Innovación y Transferencia en Tecnología Agropecuaria (INTA). Costa Rica.

3 Escuela de Ciencias Agrarias. Universidad Nacional, Costa Rica. 
mente dependientes de la asociación simbiótica, debido a la producción de raíces gruesas y con pocos pelos absorbentes (Ferrera-Cerrato y González-Chávez 1998; Alarcón y Ferrera-Cerrato 2003).

La aplicación de esta tecnología a nivel de vivero tiene ventajas económicas de gran interés ya que se pueden obtener plantas micorrizadas vigorosas en menor tiempo, con menos costos de producción y, en el caso de plantas para injertar, se puede disminuir el tiempo para alcanzar el diámetro de tallo adecuado (Sieverding 1991; Alarcón et al. 1997). Es muy importante analizar esta alternativa para los sistemas de producción en agricultura sostenible, cuyas prioridades son bajar insumos y establecer agro-ecosistemas más naturales.

Muchos estudios señalan la introducción satisfactoria de HMA seleccionados, en semilleros y materiales de desarrollo de plantas en vivero (González et al.1998; Ferrera-Cerrato y González 1998; Martínez y Melchor 2001; Alarcón y Ferrara-Cerrato 2003); también se menciona que no todas las especies y variedades de cítricos se benefician de igual forma y que son diversos los factores que afectan positiva o negativamente la micorrización (Dehne 1990).

En el país no se han estudiado hasta la fecha los posibles beneficios de esta simbiosis en cítricos, por eso se desarrolló esta investigación con el objetivo de hacer uso eficiente de la inoculación con HMA efectivos en viveros, como una opción tecnológica que ayude a conseguir plantas de calidad con una menor utilización de insumos químicos y que contribuya a la sostenibilidad del sistema. Para este estudio se consideraron dos portainjertos de cítricos: Citrus volkameriana, que es muy utilizado a nivel de viveros y Citrumelo swingle, que está entre los mejores patrones de cítricos actuales.

\section{MATERIALES Y MÉTODOS}

Se realizaron dos experimentos en el vivero Serra, ubicado en Sabanilla de Alajuela, a $1200 \mathrm{msnm}$, con una temperatura media de $22{ }^{\circ} \mathrm{C}$ y precipitación anual media de 2.500 $\mathrm{mm}$. En el primer experimento se utilizó Citrus volkameriana, portainjerto comúnmente utilizado en los viveros citrícolas, muy vigoroso, tolerante a muchas enfermedades, donde se evaluaron 24 tratamientos resultantes de la interacción de dos factores y un tratamiento adicional. El primer factor fue la desinfección del sustrato de siembra con cuatro técnicas diferentes: a) agua caliente (técnica empleada en el vivero), b) Basamid (dazomet), c) PCNB (Pentachloronitrobenzeno) y d) solarización. El segundo factor consistió de la inoculación en el semillero con hongos micorrizógenos: a) testigo sin inoculación (Sin inóculo), b) inoculado con Glomus manihotis (G. manihotis), c) población nativa de Atenas, Alajuela (P.N.), d) Glomus sp., y e) mezcla de los tres inóculos (Mezcla). Además, se incluyó un tratamiento adicional que consistió en inocular la población nativa de Atenas en el momento del transplante (Ino Trans). La población nativa de hongos micorrizógenos se obtuvo tomando suelo de la rizosfera de un banco de Cratylia argentea en la Escuela Centroamericana de Ganadería, Atenas. El suelo se colocó en macetas mezclado con suelo esterilizado en autoclave y se sembró Brachiaria decumbens como planta trampa, luego de cuatro meses se obtuvo el inoculante que consistió de raíces colonizadas, suelo con esporas y micelio. Las especies de Glomus fueron multiplicadas de igual forma en macetas con Bracharia. El sustrato se preparó tal como se utiliza en el vivero; consiste de una mezcla de suelo, granza y compost en proporción 1:0,5:0,1 en volumen respectivamente, más 20 gramos de cal. El análisis (Cuadro 1) indica que no presenta ningún problema de fertilidad. Tiene un $\mathrm{pH}$ débilmente ácido, buenos contenidos de nutrimentos y un nivel medio de $P$. 
Cuadro 1. Análisis químico del sustrato utilizado para la siembra de los árboles de cítricos en el vivero y en los experimentos. Alajuela, Costa Rica. 2003 - 2004.

\begin{tabular}{|c|c|c|c|c|c|c|c|c|c|c|}
\hline \multirow{3}{*}{$\begin{array}{l}\text { Identificación } \\
\text { Sustrato Vivero } \\
\text { Serra - Alajuela }\end{array}$} & \multicolumn{5}{|c|}{ cmol(+)/l } & \multicolumn{5}{|c|}{$\mathrm{mg} / \mathrm{l}$} \\
\hline & $\mathrm{pH}$ & $\mathrm{Al}$ & $\mathrm{Ca}$ & $\mathrm{Mg}$ & K & $P$ & $\mathrm{Zn}$ & $\mathrm{Mn}$ & $\mathrm{Cu}$ & $\mathrm{Fe}$ \\
\hline & 6 & 0,1 & 7,5 & 2,4 & 1,01 & 19 & 4,5 & 9 & 14 & 65 \\
\hline
\end{tabular}

El sustrato se dispuso en eras de $2 \mathrm{~m} \mathrm{x}$ $0,90 \mathrm{~m} \times 0,20 \mathrm{~cm}$ donde se aplicaron los tratamientos de desinfección. Con los sustratos desinfectados se llenaron bandejas de plástico de $40 \mathrm{~cm} \times 20 \mathrm{~cm} \times 0,20 \mathrm{~cm}$ de capacidad. Primero se colocó una capa de sustrato, luego de acuerdo al tratamiento, una capa de aproximadamente $0,5 \mathrm{~cm}$ de inóculo y nuevamente sustrato.

Se sembraron semillas pre-germinadas de Citrus volkameriana a dos centímetros de distancia unas de otras, las bandejas se mantuvieron en invernadero hasta que las plantitas alcanzaron una altura promedio de 15 centímetros (Figura 1). El transplante se realizó a raíz desnuda a bolsas que contenían el sustrato según el tratamiento de desinfección correspondiente. Las bolsas se colocaron a la intemperie en un diseño completamente al azar con diez repeticiones (Figura 2). Las variables medidas fueron la altura de las plantas y el diámetro del tallo en la base a los 90 y 120 días después del transplante a bolsas y, al final del experimento se midieron el peso seco de la parte aérea $\left(60^{\circ} \mathrm{C}\right)$, la concentra-

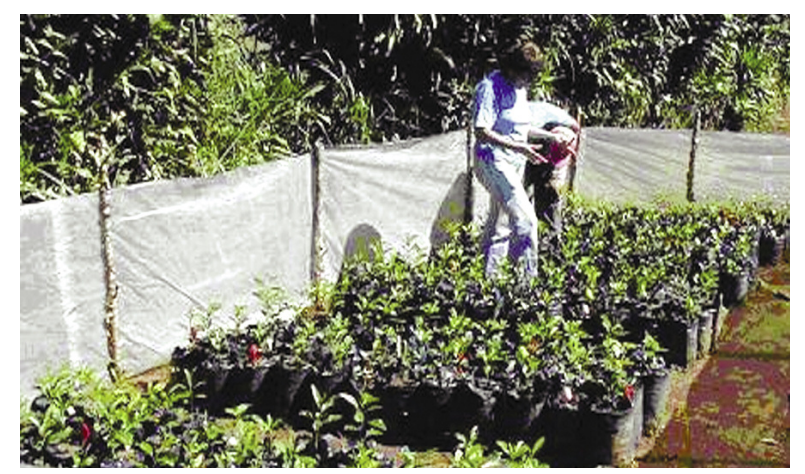

Figura 2. Vista parcial del experimento $N \ddagger 1$ : Estudio sobre desinfección de sustrato y fecha de inoculación con HMA en Citrus volkameriana (Foto tomada: 26-09-2003).

ción de nutrimentos a nivel foliar y la colonización de las raíces por hongos micorrícicos (Brundrett et al. 1996). Los análisis se realizaron en el Laboratorio de Suelos, Foliares y Aguas del INTA.

El segundo experimento se realizó con Citrumello swingle, portainjerto de uso común en los viveros, con el que se obtienen altas producciones de cítricos, resistente a la pudrición radical ocasionada por Phytophthora y

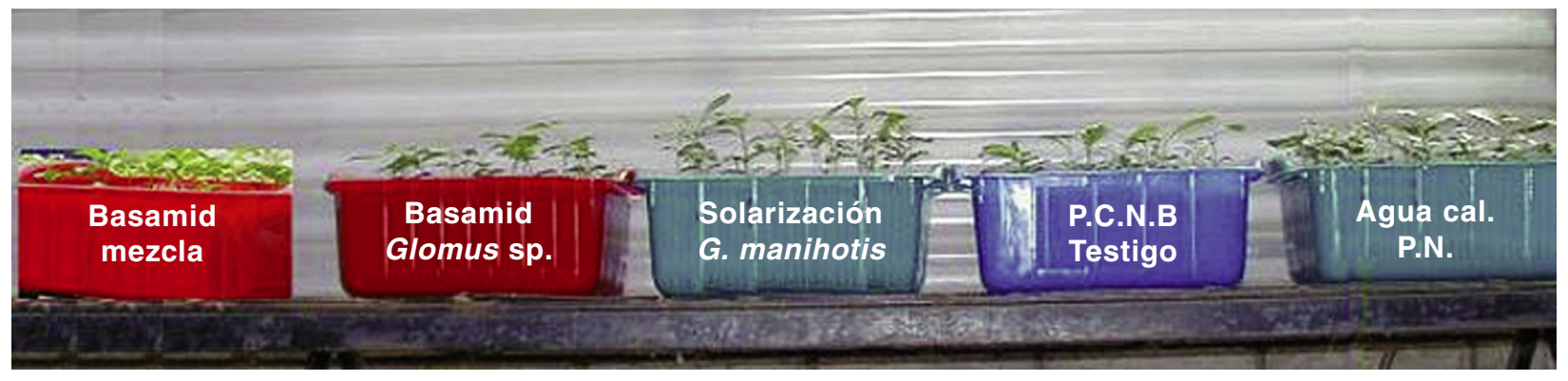

Figura 1. Vista parcial del semillero que representa los tratamientos considerados en el experimento. 
a la enfermedad de la tristeza (Figura 3). Se evaluaron seis tratamientos producto de los factores método de desinfección de sustratos y momento o época de la inoculación. El primer factor consistió de dos niveles: a) agua caliente y b) Basamid, mientras que el segundo factor se dividió en a) testigo sin inoculación (Sin inóculo), b) inoculación con la población nativa de Atenas en el momento de establecer el semillero (P.N.) y c) inoculación con la población nativa cuando se realizó el transplante a bolsas (Ino Trans). El sustrato y el semillero se manejaron tal como se anotó para el primer experimento. Las bolsas se colocaron a la intemperie en un diseño completamente al azar con diez repeticiones. Se midieron las mismas variables descritas para el primer experimento.

Los datos obtenidos se sometieron a un análisis de varianza (ANDEVA) para determinar una posible interacción entre factores o efectos simples; cuando esto último ocurrió se compararon los niveles mediante la prueba de diferencia mínima significativa (DMS).

\section{RESULTADOS Y DISCUSIÓN}

\section{1.- Resultados y discusión del experimen- to con Citrus volkameriana:}

Del análisis estadístico de los resultados obtenidos se puede inferir que los diferentes tratamientos de desinfección de sustratos tuvieron influencia en la efectividad de los HMA.

Las técnicas de desinfección naturales (agua caliente y solarización) fueron más amigables para la asociación simbiótica; el uso de agroquímicos como desinfectantes la afectaron negativamente, pero su influencia varió según el hongo utilizado como inóculo (Figura 4).

El ANDEVA reveló que la interacción "técnica de desinfección" * "Inóculación con HMA" fue significativa. El uso de PCNB como desinfectante del sustrato afectó negativamente a todos los HMA, pero en mayor medida a $G$. manihotis; su influencia fue menor cuando se usó Mezcla e Ino Trans. El uso de Basamid
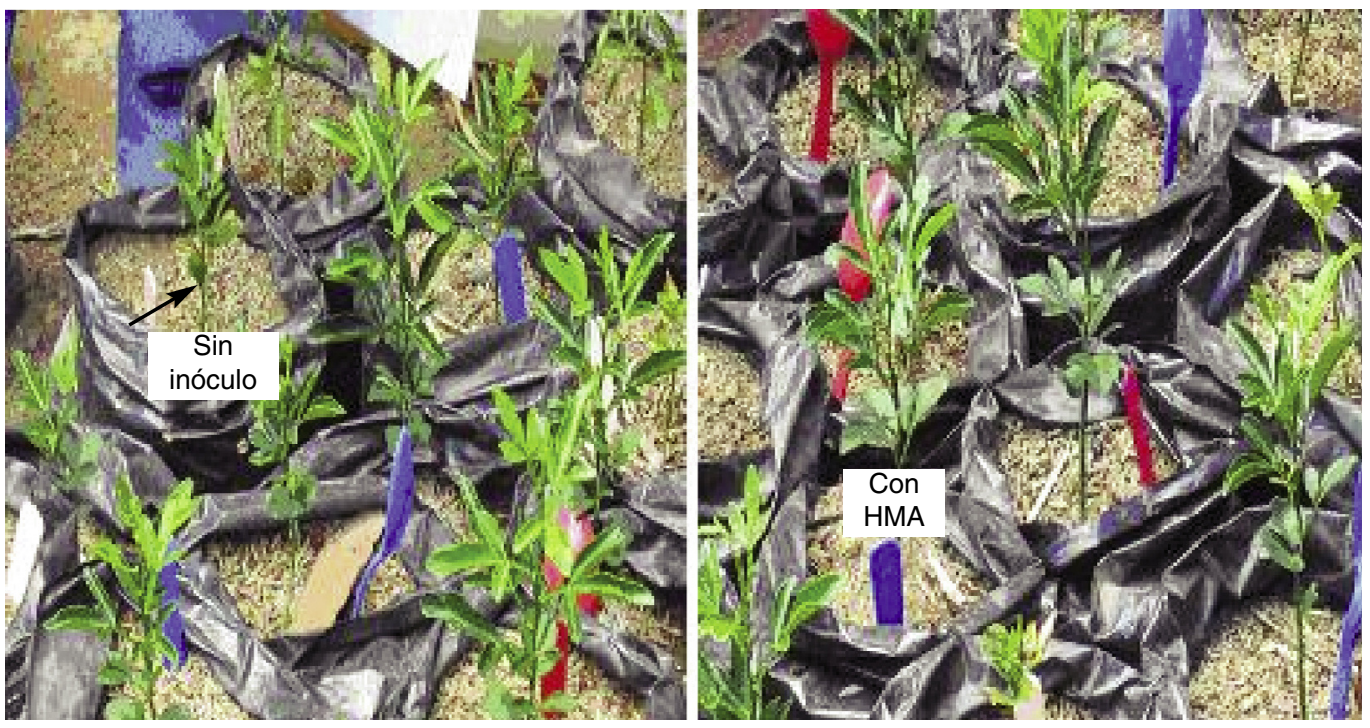

Figura 3. Vista parcial del experimento sobre desinfección de sustrato y fecha de inoculación con HMA en Citrumelo swingle. Foto tomada el 27-02-04. 


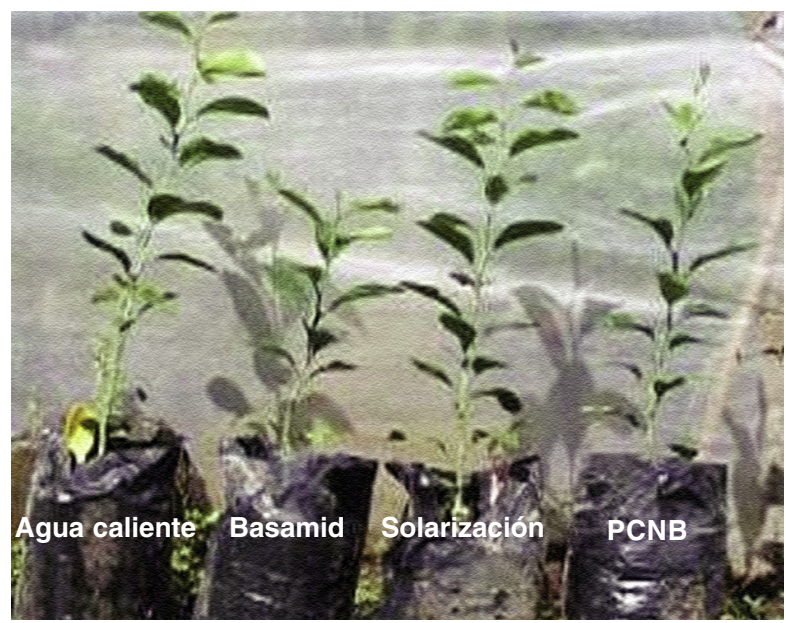

Figura 4. Arbolitos de Citrus volkameriana inoculados con P.N. de micorrizas, con cuatro tratamientos de desinfección de sustratos, a los 50 días del transplante.

como tratamiento de desinfección, perjudicó a todos los HMA introducidos como inóculos, pero en menor medida cuando se usó Ino Trans., posiblemente porque hubo un período de tiempo más prolongado entre la aplicación del desinfectante al sustrato y la inoculación con HMA.

EI ANDEVA para la variable altura (Figura 5) medida a los 90 días del transplante, indicó una interacción significativa entre los dos factores considerados en el experimento $(p=$
0,0001). En las mediciones efectuadas a los 120 días, momento en que se dio por finalizado el experimento, estas interacciones fueron perdiendo fuerza, sin embargo se siguieron manifestando $(p=0,08)$. El agua caliente no afectó la asociación simbiótica, sin embargo, el inóculo con G. manihotis no causó efecto positivo en la altura comparándolo con el tratamiento "sin inóculo", probablemente porque la micorriza nativa (presente en todos los tratamientos) pudo interactuar y disminuir el efecto de este hongo introducido, reduciendo la potencialidad del tratamiento. Los árboles inoculados con P.N. y con Glomus sp. fueron significativamente más altos que los del tratamiento "sin inóculo" y que los inoculados con otros HMA.

El sustrato tratado con agua caliente fue el que dio significativamente mayor diámetro con respecto a los otros tratamientos de desinfección (Figura 6).

En cuanto a los diferentes inóculos de HMA, se debe acotar que los arbolitos inoculados con P.N. presentaron los mayores diámetros comparados con los otros tratamientos de inóculos para todos los tratamientos de desinfección, con excepción de P.C.N.B., que afectó negativamente a esta población. Las interacciones manifestadas entre los factores

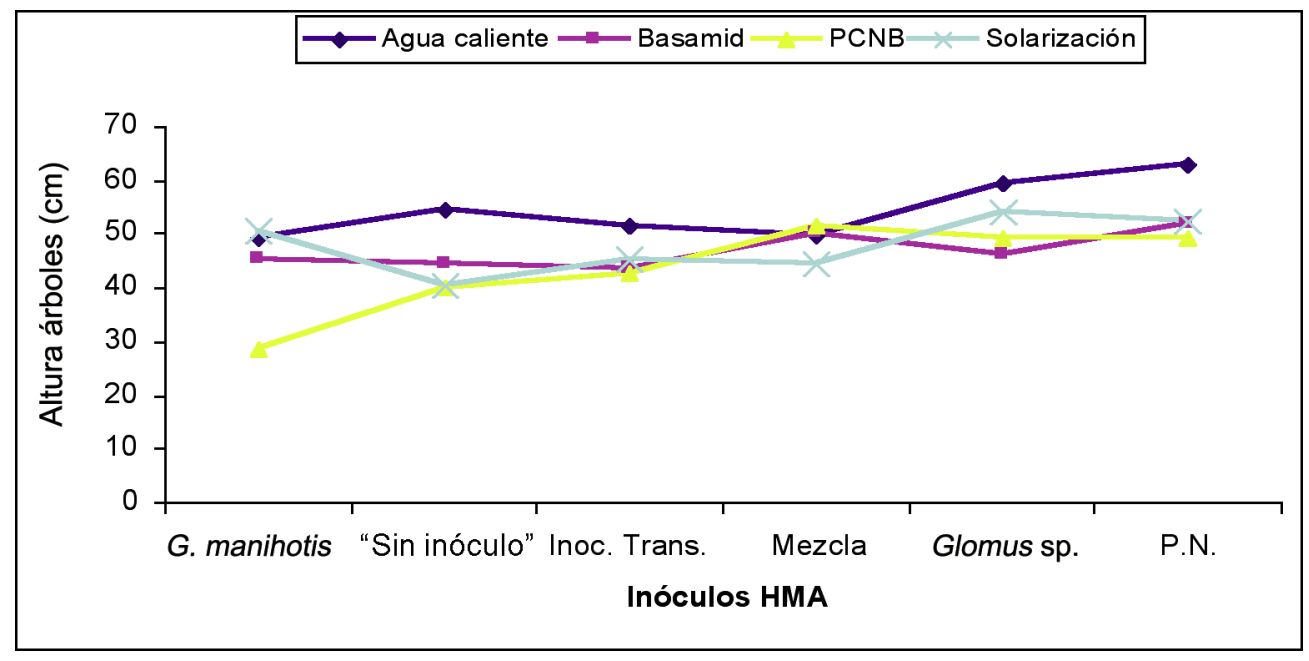

Figura 5. Interacciones en altura de los árboles de Citrus volkameriana con cuatro técnicas de desinfección del sustrato y diferentes inóculos de HMA, a los 120 días del transplante. Alajuela, Costa Rica. 2003 - 2004. 


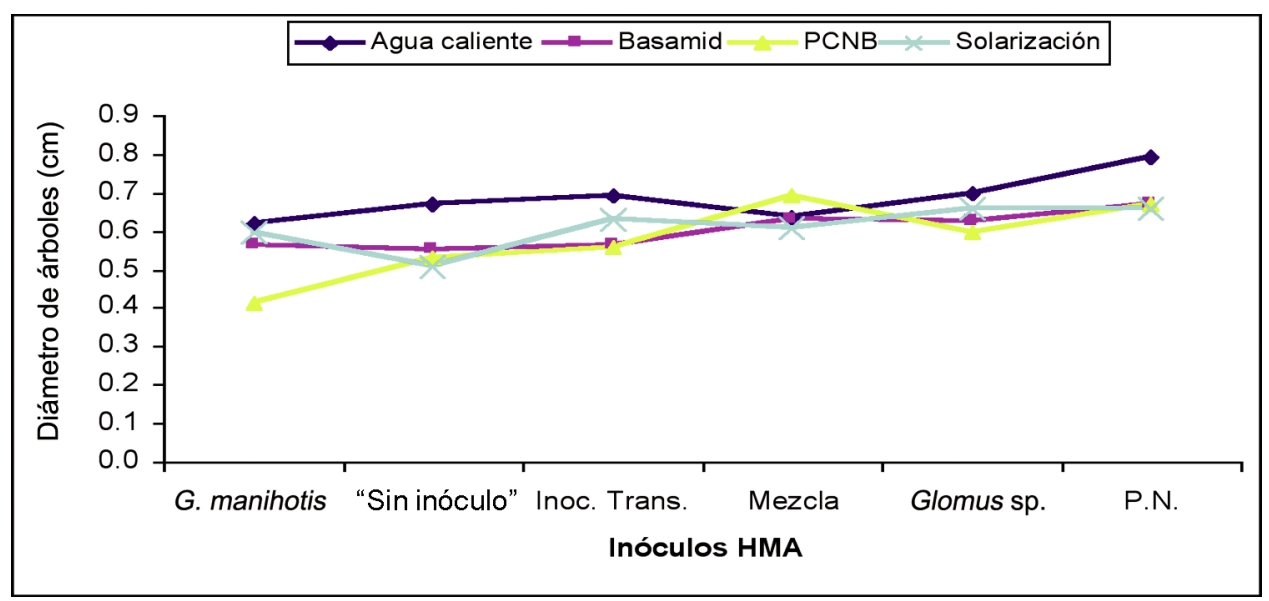

Figura 6. Interacciones en diámetro de los árboles de Citrus volkameriana con cuatro técnicas de desinfección del sustrato y diferentes inóculos de HMA, a los 120 días del transplante. Alajuela, Costa Rica. 2003 - 2004.

desinfección e inóculo, se notaron con mayor intensidad en las medidas de diámetro realizadas a los 120 días del transplante $(p=$ $0,02)$.

Las diferencias significativas encontradas en este experimento para las variables de diámetro y altura se vieron reflejadas en la biomasa aérea de los arbolitos (Figura 7) y en el porcentaje de árboles listos para injertación a los 120 días del transplante a bolsa (Figura
8). El tratamiento con agua caliente como método de desinfección, inoculado con P.N. fue significativamente el de mayor biomasa (Prueba LSD, $p \leq 0,05$ ) y las interacciones entre los factores fueron significativas $(p=$ $0,06)$.

Se había acordado previamente terminar el experimento cuando el mejor tratamiento alcanzara un porcentaje de árboles injertables de $90 \%$ (Diámetro de tallo a $0,20 \mathrm{~m}$ de la

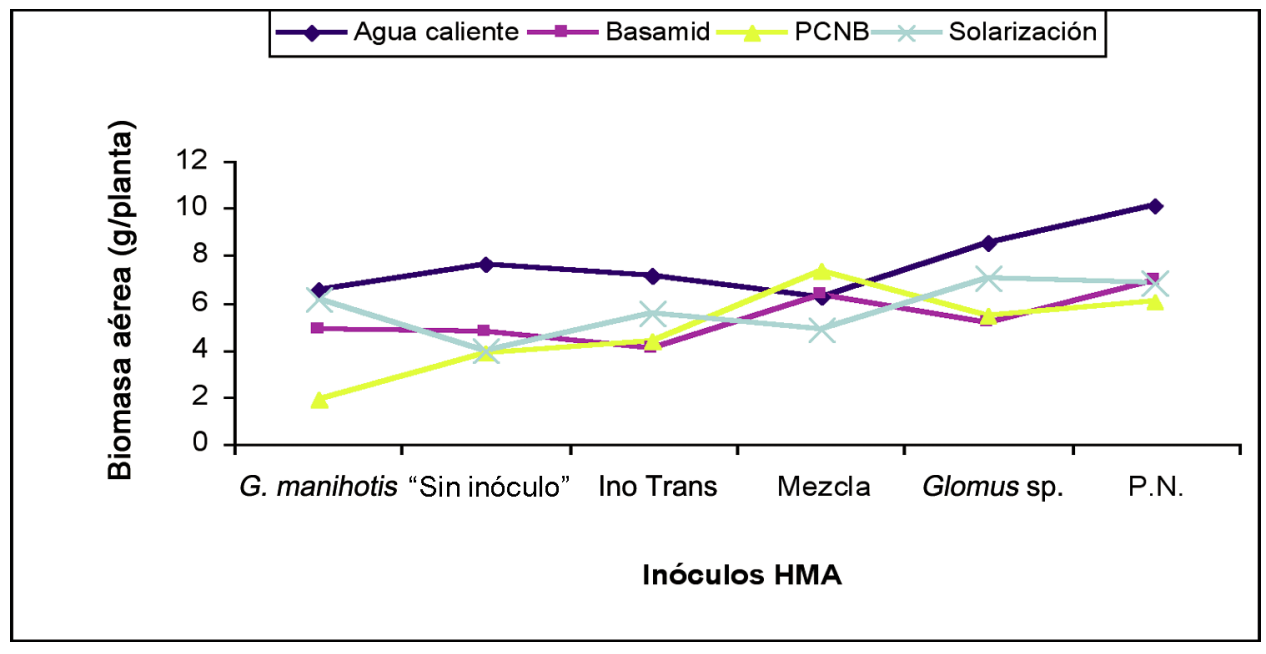

Figura 7. Interacciones en biomasa aérea de los árboles de Citrus volkameriana con cuatro técnicas de desinfección del sustrato y diferentes inóculos de HMA. Alajuela, Costa Rica. 2003 - 2004. 
口 Agua a Basamid a PCNB $\square$ Solarización

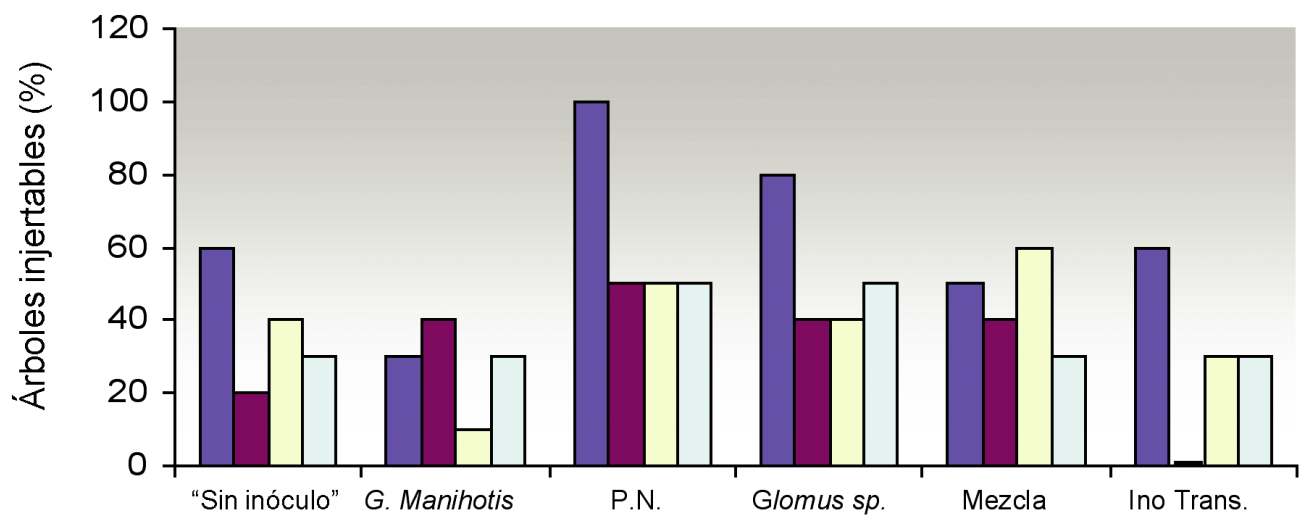

Figura 8. Porcentaje de árboles injertables a los 120 días de transplante, con cuatro tratamientos de desinfección y diferentes inóculos de HMA. Alajuela, Costa Rica. 2003 $-2004$.

base $\geq 0,60 \mathrm{~cm}$ altura aproximada $=0,50 \mathrm{~m}$ ). Esto se cumplió para el tratamiento con sustrato desinfectado con agua caliente e inoculado con P.N., a los 120 días del transplante (Figura 8). Los resultados obtenidos representan un ahorro en la permanencia de los árboles en vivero de dos a tres meses y conlleva al uso de menos insumos y una mayor relación beneficio costo. También se debe hacer referencia a la uniformidad que alcanzaron estos árboles, cualidad valiosa en viveros.

Diversos trabajos reconocen los efectos positivos que ejerce la asociación simbiótica en los cítricos (Alarcón et al. 1996; FerreraCerrato y González 1993; Sierverding 1991); sin embargo, la respuesta depende de factores muy diversos y no todas las especies de cítricos se benefician de igual forma (Dehne 1990). Alarcón y Ferrara-Cerrato (2003) señalan el carácter micotrófico de Citrus volkameriana, aún en presencia de buenas cantidades de $\mathrm{P}$ (fósforo). Ellos concluyen que si se utiliza un sustrato con buenas condiciones de fertilidad, la sola inoculación con micorrizas produce efectos significativos en el crecimiento de este portainjerto, por lo que la fertilización resultaría en un gasto innecesario. Koide y Kabir (2000) afirman que el micelio externo de las micorrizas es capaz de solubilizar y utilizar compuestos orgánicos de $\mathrm{P}$ presentes en los sustratos enriquecidos con abonos orgánicos.

La colonización de raíces por HMA fue afectada significativamente cuando se usó basamid como tratamiento para desinfección de sustrato. Los otros tratamientos de desinfección no tuvieron influencia en el porcentaje de colonización. Si se comparan los HMA, en los tratamientos con Glomus sp., P.N. e Ino Trans, se dieron los mayores porcentajes de colonización de raíces, comparándolos con el tratamiento "sin inóculo" y con G. manihotis. El tratamiento Mezcla dio resultados intermedios (Figura 9).

No se encontraron diferencias en el contenido porcentual de nutrimentos de la parte aérea (Cuadro 2). Si la expresión de los resultados se lleva a absorción neta de nutrimentos, sí se manifiestan diferencias significativas, debido a la biomasa característica de cada tratamiento.

\section{2.- Resultados y discusión del experimen- to con Citrumelo swingle}

El método de desinfección de sustrato con basamid afectó negativamente la altura, el diámetro y la biomasa de Citrumelo swingle 


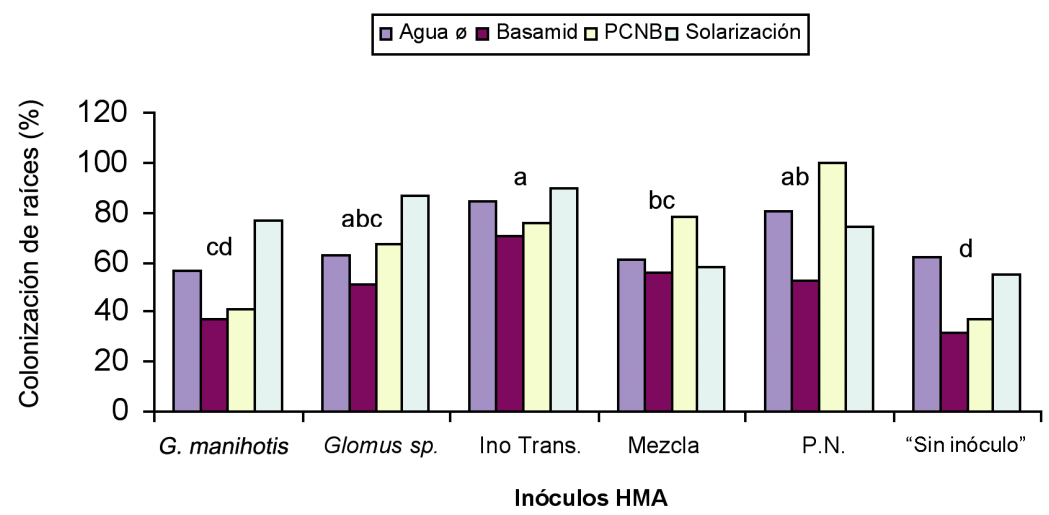

Figura 9. Colonización de raíces de Citrus volkameriana por HMA, en sustrato de vivero con diferentes tratamientos de desinfección e inoculación. Alajuela, Costa Rica. 2003 - 2004.

${ }^{*}$ Letras distintas indican diferencias significativas $(p \leq 0,05)$.

(Cuadro 3). Las diferencias fueron más notorias en las primeras medidas realizadas, a los 130 días del transplante. Sin embargo, estas diferencias se mantuvieron a través del desarrollo del experimento y fueron significativas en las medidas realizadas al término del mismo, a los 180 días del transplante.

Corroborando los resultados obtenidos con Citrus volkameriana, la técnica de desinfección

Cuadro 2. Análisis de nutrimentos de la parte aérea de Citrus volkameriana, con diferentes tratamientos de desinfección de sustrato e inoculación con HMA. Alajuela, Costa Rica. 2003 - 2004.

\begin{tabular}{|c|c|c|c|c|c|c|c|c|c|}
\hline \multirow{2}{*}{$\begin{array}{l}\text { Identificación } \\
\text { Tratamiento* }^{*}\end{array}$} & \multicolumn{6}{|c|}{$\%$} & \multicolumn{3}{|c|}{$\mathrm{mg} / \mathbf{k g}$} \\
\hline & $\mathbf{N}$ & $\mathbf{P}$ & $\mathbf{k}$ & $\mathrm{Ca}$ & Mg & $\mathrm{Cu}$ & Zn & Mn & $\mathrm{Fe}$ \\
\hline Agua $\theta, G$ manihotis & 3,0 & 0,23 & 1,58 & 1,61 & 0,14 & 16 & 20 & 58 & 639 \\
\hline Agua $\theta$, Glomus sp. & 2,9 & 0,21 & 1,45 & 1,73 & 0,13 & 12 & 15 & 47 & 528 \\
\hline Agua $\theta$, Ino Trans & 3,3 & 0,21 & 1,50 & 1,76 & 0,14 & 13 & 15 & 50 & 453 \\
\hline Agua $\theta$, Mezcla & 3,2 & 0,21 & 1,49 & 1,71 & 0,13 & 13 & 13 & 42 & 420 \\
\hline Agua $\theta$, P.N. & 3,1 & 0,21 & 1,59 & 1,65 & 0,12 & 13 & 15 & 43 & 428 \\
\hline Agua $\theta$, Sin inóculo & 3,1 & 0,21 & 1,51 & 1,63 & 0,13 & 13 & 13 & 46 & 443 \\
\hline Basamid, G manihotis & 3,4 & 0,22 & 1,54 & 1,79 & 0,14 & 12 & 13 & 47 & 380 \\
\hline Basamid, Glomus sp. & 3,4 & 0,23 & 1,48 & 1,74 & 0,14 & 12 & 12 & 45 & 318 \\
\hline Basamid, Ino Trans & 3,4 & 0,22 & 1,34 & 1,71 & 0,14 & 13 & 11 & 45 & 349 \\
\hline Basamid, Mezcla & 3,1 & 0,21 & 1,38 & 1,66 & 0,14 & 10 & 12 & 46 & 297 \\
\hline Basamid, P.N. & 3,2 & 0,21 & 1,33 & 1,70 & 0,12 & 12 & 14 & 55 & 325 \\
\hline Basamid, Sin inóculo & 3,2 & 0,23 & 1,47 & 1,67 & 0,13 & 11 & 11 & 44 & 288 \\
\hline P.C.N.B., G manihotis & 3,5 & 0,23 & 1,60 & 1,75 & 0,14 & 12 & 14 & 48 & 312 \\
\hline P.C.N.B., Glomus sp. & 3,2 & 0,22 & 1,69 & 1,77 & 0,12 & 12 & 14 & 46 & 370 \\
\hline P.C.N.B., Ino Trans & 3,3 & 0,21 & 1,50 & 1,71 & 0,12 & 13 & 10 & 44 & 286 \\
\hline P.C.N.B., Mezcla & 3,1 & 0,21 & 1,78 & 1,66 & 0,11 & 12 & 12 & 43 & 262 \\
\hline P.C.N.B., P.N. & 3,3 & 0,21 & 1,63 & 1,70 & 0,11 & 13 & 13 & 42 & 316 \\
\hline P.C.N.B., Sin inóculo & 3,4 & 0,22 & 1,56 & 1,67 & 0,11 & 12 & 13 & 44 & 320 \\
\hline Solarización, G manihotis & 3,1 & 0,21 & 1,39 & 1,64 & 0,11 & 11 & 12 & 43 & 349 \\
\hline Solarización, Glomus sp. & 2,9 & 0,21 & 1,31 & 1,55 & 0,12 & 10 & 9 & 38 & 195 \\
\hline Solarización, Ino Trans & 3,2 & 0,21 & 1,33 & 1,57 & 0,12 & 13 & 15 & 41 & 307 \\
\hline Solarización, Mezcla & 3,2 & 0,21 & 1,29 & 1,52 & 0,13 & 11 & 14 & 44 & 219 \\
\hline Solarización, P.N. & 3,1 & 0,21 & 1,49 & 1,60 & 0,12 & 11 & 12 & 44 & 292 \\
\hline Solarización, Sin inóculo & 3,1 & 0,23 & 1,76 & 1,69 & 0,13 & 12 & 16 & 45 & 300 \\
\hline
\end{tabular}

${ }^{\star}$ Agua $\theta=$ agua caliente. 
Cuadro 3. Medidas promedio de altura, diámetro, biomasa aérea y biomasa de raíces de Citrumelo swingle con diferentes técnicas de desinfección de sustrato y tres tratamientos de inoculación con HMA. Factor desinfección. Alajuela, Costa Rica. 2003 - 2004.

\begin{tabular}{lcccc}
\hline $\begin{array}{l}\text { Desinfección } \\
\text { de sustrato }\end{array}$ & $\begin{array}{c}\text { Altura* } \\
\text { (cm) }\end{array}$ & $\begin{array}{c}\text { Diámetro* } \\
\text { (cm) }\end{array}$ & $\begin{array}{c}\text { Biomasa } \\
\text { aérea* } \\
\text { (g/planta) }\end{array}$ & $\begin{array}{c}\text { Biomasa } \\
\text { raíces* } \\
\text { (g/planta) }\end{array}$ \\
\hline Basamid & $39,0 \mathrm{~b}$ & $0,69 \mathrm{~b}$ & $3,76 \mathrm{~b}$ & $2,96 \mathrm{~b}$ \\
Agua caliente & $44,9 \mathrm{a}$ & $0,82 \mathrm{a}$ & $5,27 \mathrm{a}$ & $3,91 \mathrm{a}$ \\
\hline
\end{tabular}

* Letras distintas indican diferencias significativas $(p<=$ $0,05)$.

de sustratos que emplea agua caliente fue la más amigable con la asociación simbiótica (diferencia de altura entre tratamientos de desinfección: Figura 10).

Cuando se desinfectó con Basamid, los dos tratamientos de inoculación con HMA (P.N. e Ino Trans) produjeron los mayores incrementos en la altura y diámetro de los arbolitos comparados con el testigo sin inóculo; no hubo diferencias significativas entre las fechas de aplicación del inóculo, aunque sí se manifestó una tendencia positiva con P.N. (Cuadro 4).

Cuando se usó el agua caliente como método de desinfección se obtuvieron los mejores resultados. La biomasa aérea y de raíces

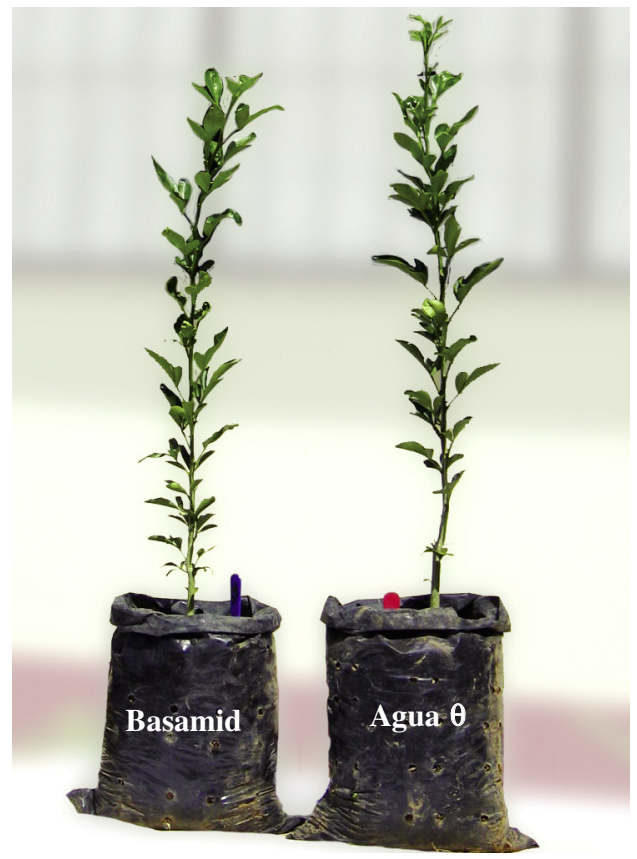

Figura 10. Citrumelo swingle inoculado con población nativa de HMA (P.N.) y con dos tratamientos de desinfección de sustrato.

presentaron diferencias significativas en el tratamiento donde se combinó el agua caliente con inóculo de P.N, cotejadas con Ino Trans y el tratamiento sin inóculo (Figura 11).

En un estudio anterior Schweizer y Salas (2002) no encontraron diferencias significativas para Citrumelo swingle inoculado, o no,

Cuadro 4. Medidas de altura, diámetro, biomasa aérea y biomasa de raíces de Citrumelo swingle con diferentes técnicas de desinfección de sustrato y tres tratamientos de inoculación con HMA. Alajuela, Costa Rica. 2003 - 2004.

\begin{tabular}{|c|c|c|c|c|c|}
\hline $\begin{array}{l}\text { Técnicas de } \\
\text { desinfección }\end{array}$ & $\begin{array}{l}\text { Inoculación* } \\
\text { con HMA }\end{array}$ & $\begin{array}{l}\text { Altura* } \\
\text { (cm) }\end{array}$ & $\begin{array}{l}\text { Diámetro* } \\
\text { (cm) }\end{array}$ & $\begin{array}{c}\text { Biomasa aérea* } \\
\text { (g/planta) }\end{array}$ & $\begin{array}{c}\text { Biomasa raíces* } \\
\text { (g/ Planta) }\end{array}$ \\
\hline \multirow[t]{3}{*}{ Basamid } & Sin inóculo & $27,90 \mathrm{c}$ & $0,55 \mathrm{c}$ & $1,88 d$ & $1,60 \mathrm{c}$ \\
\hline & Ino Trans & $42,07 a b$ & $0,72 \mathrm{~b}$ & $4,38 \mathrm{bc}$ & $3,48 \mathrm{~b}$ \\
\hline & P.N. & $47,10 a b$ & $0,81 a b$ & $5,03 \mathrm{bc}$ & $3,80 \mathrm{~b}$ \\
\hline \multirow[t]{3}{*}{ Agua caliente } & Sin inóculo & $38,47 \mathrm{~b}$ & $0,71 \mathrm{~b}$ & $3,57 \mathrm{c}$ & $2,80 \mathrm{bc}$ \\
\hline & Ino Trans & $46,29 a b$ & $0,85 a$ & $5,39 \mathrm{~b}$ & $3,73 \mathrm{~b}$ \\
\hline & P.N. & $49,89 \mathrm{a}$ & $0,90 \mathrm{a}$ & $6,84 \mathrm{a}$ & $5,20 \mathrm{a}$ \\
\hline DMS & & 9,8 & 0,12 & 1,46 & 1,31 \\
\hline \multicolumn{6}{|l|}{ Significancia } \\
\hline Desinfección & & 0,04 & 0,0005 & 0,0008 & 0,01 \\
\hline HMA & & 0,0002 & 0,0001 & 0,0001 & 0,0002 \\
\hline Desinfección * & HMA & ns & ns & ns & ns \\
\hline
\end{tabular}

* Letras distintas indican diferencias significativas $(p<=0,05)$ 


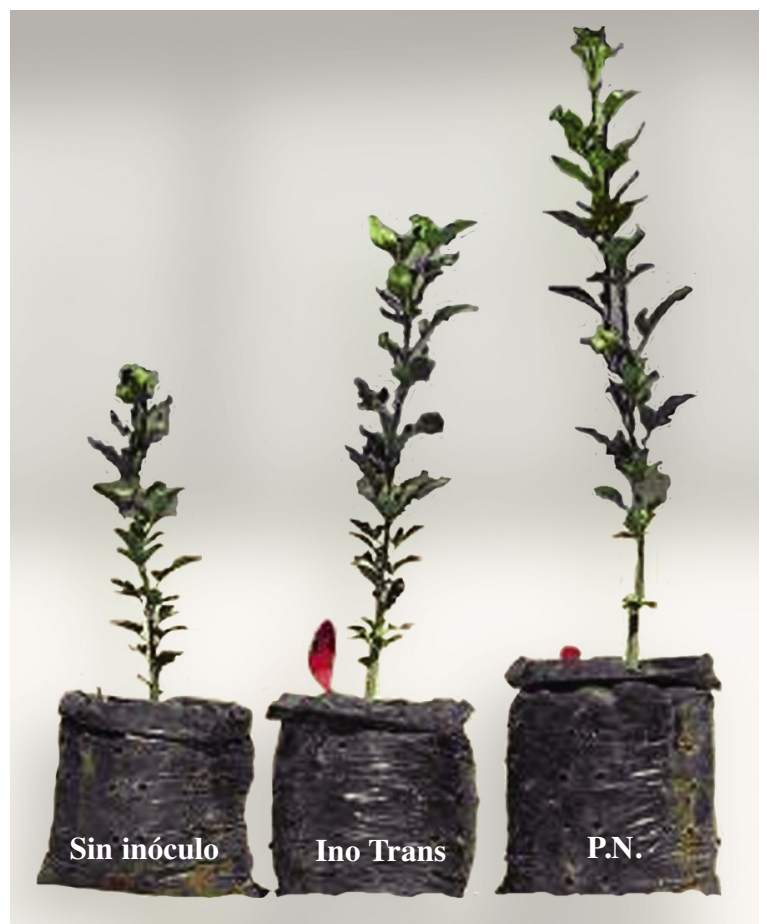

Figura 11. Citrumelo swigle en sustrato desinfectado con agua caliente y tres tratamientos de inoculación con HMA.

con HMA; sin embargo encontraron que había una tendencia positiva en el desarrollo de este portainjerto inducida por las micorrizas inoculadas al transplante de los arbolitos a bolsa. Martínez y Melchor (2001) encontraron mayor tasa de crecimiento y producción de biomasa en árboles de Citrumelo en la fase de vivero, cuando fueron inoculados con micorrizas.

En el contenido porcentual de nutrimentos no se encontraron diferencias entre los trata- mientos (Cuadro 5). Como en el caso de Citrus volkameriana, si se analiza la absorción total de nutrimentos, sí hay diferencias significativas entre los diferentes tratamientos.

\section{CONCLUSIONES Y RECOMENDACIONES}

- La técnica de desinfección de sustrato que emplea agua caliente fue la más amigable para la asociación simbiótica portainjertos de cítricos - HMA.

- El uso de Basamid para desinfectar el sustrato de vivero, afectó negativamente el desarrollo de los HMA y de la asociación simbiótica.

- El inóculo con población de micorrizas nativas incrementó el diámetro, la altura y la biomasa de Citrus volkameriana y Citrumelo swingle.

- Con la incorporación de micorrizas nativas se acortó el tiempo a injertación en 60 días o más en los dos portainjertos y con esto se redujeron los costos de permanencia de las plantas en el vivero en más del $20 \%$ para ambos casos.

- Se recomienda realizar la inoculación de HMA desde el establecimiento del semillero para facilitar la labor de inoculación y disminuir la cantidad de inóculo necesario; y porque esta técnica de inoculación

Cuadro 5. Contenido de nutrimentos de la parte aérea de Citrumelo swingle, con diferentes tratamientos de desinfección de sustrato e inoculación con HMA. Alajuela, Costa Rica. 2003 - 2004.

\begin{tabular}{|c|c|c|c|c|c|c|c|c|c|}
\hline \multirow{2}{*}{$\begin{array}{l}\text { Identificación } \\
\text { Tratamientos* }\end{array}$} & \multicolumn{5}{|c|}{$\%$} & \multicolumn{4}{|c|}{ mg/kg } \\
\hline & $\mathbf{N}$ & $\mathbf{P}$ & $\mathbf{k}$ & $\mathrm{Ca}$ & Mg & $\mathrm{Cu}$ & $\mathrm{Zn}$ & $M n$ & $\mathrm{Fe}$ \\
\hline Agua $\theta$, Ino Trans & 2,1 & 0,20 & 1,21 & 1,22 & 0,21 & 18,8 & 18,6 & 52,1 & 189 \\
\hline Agua $\theta$, P.N. & 1,9 & 0,20 & 1,25 & 1,30 & 0,20 & 16,7 & 18,9 & 36,9 & 156 \\
\hline Agua $\theta$, Sin inóculo & 2,2 & 0,21 & 1,81 & 1,25 & 0,30 & 40,0 & 30,7 & 66,3 & 301 \\
\hline Basamid, Ino Trans & 1,8 & 0,18 & 1,16 & 1,12 & 0,19 & 14,1 & 20,8 & 89,8 & 220 \\
\hline Basamid, P.N. & 1,8 & 0,18 & 1,28 & 1,14 & 0,20 & 18,8 & 18,9 & 82,8 & 242 \\
\hline Basamid, Sin inóculo & 1,8 & 0,18 & 1,19 & 1,17 & 0,21 & 11,7 & 17,5 & 69,5 & 304 \\
\hline
\end{tabular}

${ }^{*}$ Agua $\theta=$ agua caliente. 
fue la que incrementó en mayor medida el desarrollo de los portainjertos Citrus volkameriana y Citrumelo swingle.

\section{AGRADECIMIENTOS}

A FITTACORI por la ayuda económica; a su Directora, Ing. Guadalupe Gutiérrez y al personal, por su esmerada atención. Al Ing. Ramón L. Hernández y a toda la gente del vivero SERRA. Al Ing. Alexis Vargas y al personal del Laboratorio de Suelos, Foliares y Aguas del INTA. A los técnicos Sergio Rodríguez y Jorge Vásquez por su valiosa ayuda. A la Bach. Q. Paola Fuentes por su valioso aporte en la revisión del trabajo.

\section{LITERATURA CITADA}

Alarcón A; Ferrera-Cerrato R. 1999. Manejo de la micorriza arbuscular en sistemas de propagación de plantas frutícolas. Terra 17: 179191.

2003. Aplicación de fósforo e inoculación de hongos micorrózicos arbusculares en el crecimiento y estado nutricional de Citrus volkameriana Tan \& Pasq. Terra 21 (1): 91-99.

Alarcón, A.; Ferrera-Cerrato, R.; Villegas Monter, A.; González-Chávez M. C.; Almaraz-Suárez, J. J. 1996. Respuesta del portainjerto Citrus volkameriana tolerante al virus de la tristeza, a la inoculación endomicorrícica. I Simposium Nacional de la Simbiosis Micorrícica. Universidad Veracruzana y Colegio de Postgraduados, Xalapa, p. 32.

Alarcón, A.; Ferrera-Cerrato, R.; Villegas Monter, A.; Almaraz-Suárez, J J. 1997. Efecto de la simbiosis micorrícica en naranja valencia injertada en Citrus volkameriana. In: Ordaz Chaparro, V. M.; G. Alcantar G.; C. Castro B.; M. Mejía P. (eds.). Memoria del XXVIII Congreso Nacional de la Ciencia del Suelo. p. 148.

Brundrett, M.; Bougher, N.; Dell, B.; Grove, T.; Malajczuk, N. 1996. Working with mycorrhizas in forestry and agriculture. Australian Centre for International Agricultural Research (ACIAR) Monograph 32, $374 \mathrm{p}$.
Chacón, AM, Cuenca, G. 1998. Efecto de las micorrizas arbusculares y de la fertilización con fósforo, sobre el crecimiento de la guayaba en condiciones de vivero. Agronomía Tropical 48(4):425-440.

Dehne, H. W. 1990. Application of inocula of VA mycorrhizal fungi in inorganic carrier materials in practical agriculture and horticulture. Eighth North American Conference on Mycorrhizae. Jackson, Wyoming. 1990. p. 73.

Ferrera-Cerrato, R.; González M. C. 1993. Dinámica de crecimiento de cítricos bajo el efecto de la inoculación de hongos endomicorrícicos VA y niveles de fertilización fosfatada. XVII Congreso Latinoamericano de la Ciencia del Suelo. Salamanca, España. p. 448453.

1994. Bioproducción de frutales a nivel de vivero. $1^{a}$ Reunión Internacional de frutales nativos e introducidos con demanda nacional e internacional. Montecillo, Estado de México. p. 206-222.

1998. La simbiosis micorrícica en el manejo de los viveros de cítricos. In: FerreraCerrato, R.; Pérez-Moreno, J. eds. Manejo de agrosistemas sostenibles: 37-63. Textos Universitarios. Universidad Veracruzana. México. sp.

González Chavez, C.; Ferrera-Cerrato, R.; Pérez Moreno, J. 1998. Biotecnología de la micorriza arbuscular en fruticultura. Universidad Autónoma de Tlaxcala y Colegio de Postgraduados. Montecillo, Estado de México. 131 p.

Hernández, S. 2000. Situación actual de los cítricos en Costa Rica. Gerencia Programa Nacional de Cítricos. Ministerio de Agricultura y Ganadería. p. 2-5.

Jaen, C. D.; Ferrera-Cerrato, R. 1989. Efecto de la inoculación de cuatro cepas de hongos endomicorrícicos y tres niveles de fertilización NPK en zapote blanco (Casimiroa edulis) cultivada en vivero. XVI Congreso Nacional de Fitopatología. 24-26 de Julio. Montecillo, Estado de México. sp. 
Koide T; Kabir Z. 2000. Extraradical hyphae of the mycorrhizal fungus Glomus intraradices can hydrolise organic phosphate. New Phytol. 148: 511-517.

Martínez-Lara, E.; Melchor-Marroquín, J. 2001. Evaluación inicial de endomicorrizas en patrones de cítricos tolerantes a VTC, en Tlapacoyán, Veracruz. In: Memorias décimo cuarta Reunión Científica Tecnología Forestal y Agropecuaria, Veracruz, México. sp.
Schweizer, S.; Salas, E. 2002. Estudio sobre asociaciones de hongos formadores de micorrizas (HMA) en portainjertos de cítricos. Informe Final Proyecto código FE 01NM 401-6-02. Archivos Técnicos INTA. sp.

Sieverding, E. 1991. Vesicular - arbuscular mycorrhiza management in tropical agrosystems. Technical Cooperation - Federal Republic of Germany. Eschborn. sp. 